\title{
Ultraviolet Stimulated Emission from Sol-Gel Spin Coated ZnO Thin Films
}

\author{
Ahmed S. Razeen, ${ }^{1}$ A.-S. Gadallah, ${ }^{1}$ and M. M. El-Nahass ${ }^{2}$ \\ ${ }^{1}$ National Institute of Laser Enhanced Science, Laser Sciences and Interactions, Cairo University, Giza, Egypt \\ ${ }^{2}$ Faculty of Education, Physics Department, Ain Shams University, Roxy, Cairo, Egypt \\ Correspondence should be addressed to A.-S. Gadallah; agadallah@niles.edu.eg
}

Received 12 February 2017; Revised 7 March 2017; Accepted 19 March 2017; Published 27 March 2017

Academic Editor: Mikhael Bechelany

Copyright (C) 2017 Ahmed S. Razeen et al. This is an open access article distributed under the Creative Commons Attribution License, which permits unrestricted use, distribution, and reproduction in any medium, provided the original work is properly cited.

\begin{abstract}
Low cost ultraviolet stimulated emission has been generated using optical excitation of $\mathrm{ZnO}$ thin films deposited by sol-gel spin coating on $\mathrm{n}^{+}$As-doped $\langle 100\rangle \mathrm{Si}$-substrate. The number of deposited layers and the heat treatment have been investigated to obtain a film that can generate stimulated emission under optical excitation. The optimum condition for preparation of the film has been presented. X-ray diffraction and scanning electron microscope have been used for structural and morphological investigations. Input-output intensity dependence and spectral width, peak emission wavelength, and the quantum efficiency versus the pump intensity have been presented. A quantum efficiency of about $24.2 \%$ has been reported, a power exponent higher than 8 has been obtained in input-output intensity dependence, and a threshold of about $23 \mathrm{Mw} / \mathrm{cm}^{2}$ has been evaluated for the samples. The mechanism by which stimulated emission occurs has been discussed. The results show that sol-gel spin coating is a promising method for generating ultraviolet stimulated emission from $\mathrm{ZnO}$ thin films.
\end{abstract}

\section{Introduction}

Applications of ultraviolet stimulated emission have drawn the attention of researchers worldwide. This is due to the wide range of applications of this radiation in different fields. In medicine it is used in UV laser surgery [1], in therapy [2], in dermatology, such as pigmented legion and hair removal, skin rejuvenation, and scalp psoriasis [3], in dentistry, such as near-UV laser treatment of extrinsic dental enamel stains [4], in optoelectronics applications [5], such as data storage [6] and telecommunications [7], in earth science [8], and in others.

Materials and methods of preparations for films that can generate such radiation are under focus for many researchers. An appealing material that is used to generate such radiation is $\mathrm{ZnO}$. The interesting attributes of this material are abundance in nature, benign material, high transparency in visible region, wide range of electrical resistivity based on the preparation method, and high quantum yield. Such properties of $\mathrm{ZnO}$ material render it interesting for academia as well as practical applications. There are different methods for preparation of $\mathrm{ZnO}$ thin films. These methods include pulsed laser deposition [9], sputtering [10], sol-gel spin coating $[11,12]$, chemical bath deposition [13], spray pyrolysis [14], molecular beam epitaxy [15], and metal organic chemical vapor deposition [16].

There are several mechanisms by which stimulated emission is generated in $\mathrm{ZnO}$ thin films. These mechanisms include random lasing [17-19], exciton-exciton P-band lasing [20, 21], electron-hole plasma lasing or N-band lasing [22], nanosized or microsized whispering gallery mode dielectric resonators [23-26], coherent scattering of light by surface undulations [27], N-band lasing enhanced by top-down microdisc structuring [28], and coherent reflection from selfassembled $\mathrm{ZnO}$ hexagons [20].

Here, room temperature exciton-exciton lasing using solgel spin coating has been presented and theoretically analyzed for the first time, to the best of our knowledge. The method shows appealing advantages such as low cost, safety, and simplicity. Optimum condition for preparation of the films has been reported. Structural and morphological investigations for the films have been done by X-ray diffraction pattern and 
scanning electron microscope images. For stimulated emission properties, input-output intensity dependence, peak emission wavelength versus input intensity, and the spectral width versus input intensity have been presented. From the investigations of the properties of the stimulated emission, the mechanism responsible for stimulated emission has been proposed.

\section{Sample Preparation}

Materials used for the preparation of the $\mathrm{ZnO}$ thin films are zinc acetate dihydrate, $\left(\mathrm{CH}_{3} \mathrm{COO}\right)_{2} \mathrm{Zn} \cdot 2 \mathrm{H}_{2} \mathrm{O}(\mathrm{ZAD})$, diethanolamine $\left(\mathrm{NH}\left(\mathrm{CH}_{2} \mathrm{CH}_{2} \mathrm{OH}\right)_{2}\right)$, and 2-propanol, which were purchased from Oxford Laboratory Reagent, Scharlau, and Piochem, respectively. All chemicals have been utilized without further purification. The substrate that has been used is $\mathrm{n}^{+}$As-doped $\langle 100\rangle \mathrm{Si}$-substrate. The spin coater (model Spin-1200D, MIDAS system) has been utilized to deposit the films.

The procedures used to prepare $\mathrm{ZnO}$ thin films were reported elsewhere. Briefly, ZAD, 2-propanol, and diethanolamine were used as a zinc precursor, solvent, and additive, respectively. DEA improves the solubility of ZAD and stabilizes the sol. The molar concentration of ZAD was $0.75 \mathrm{M}$ and that of DEA was $0.75 \mathrm{M}$. ZAD was dissolved in 2-propanol under magnetic stirring and temperature of $50^{\circ} \mathrm{C}$ till clear solution was formed. The solution was left for $72 \mathrm{~h}$ before deposition. Two samples have been prepared, one with 19 layers (called S1) and the other with 30 layers (called S2). For silicon substrate cleaning, it was immersed in hot acetone, then hot ethanol, and then distilled water. The substrate is then dried. The first 10 layers were deposited with spin speed of $5000 \mathrm{rpm}$, and spin time of $60 \mathrm{~s}$. After each deposition, the samples were placed at a hotplate with temperature of $350^{\circ} \mathrm{C}$ for 20 minutes and then they were allowed to cool down to room temperature. Then the samples were heated at a temperature of $575^{\circ} \mathrm{C}$ for one hour. The ramp rate for this process was $8^{\circ} \mathrm{C} / \mathrm{min}$. Then another nine layers were deposited with the same conditions. Then, the samples were treated at $950^{\circ} \mathrm{C}$ for 1 hour. For S2, additional 11 layers were deposited with the same conditions. Afterwards, S2 was treated at a temperature of $950^{\circ} \mathrm{C}$ for one hour. The ramp rate was $8^{\circ} \mathrm{C} / \mathrm{min}$.

For structural characterization, X-ray diffraction measurements were recorded using Philips (PW1710 BASED) diffractometer. Scanning electron microscope, model Quanta 250 FEG, was used to capture the surface morphology of the films. For investigating emission, N2 laser at $337 \mathrm{~nm}$ was focused on the sample surface and the emission was collected by optical fiber and entered the slit of the spectrograph (StellarNet EPP2000). For investigating input-output power dependence, density filters were used to change the pump power incident on the sample.

\section{Results and Discussion}

XRD results of S1 and S2 are shown in Figure 1. The results show that the samples are polycrystalline with hexagonal structure of $\mathrm{ZnO}$ (JCPDS card 79-0205). S1 has the strongest

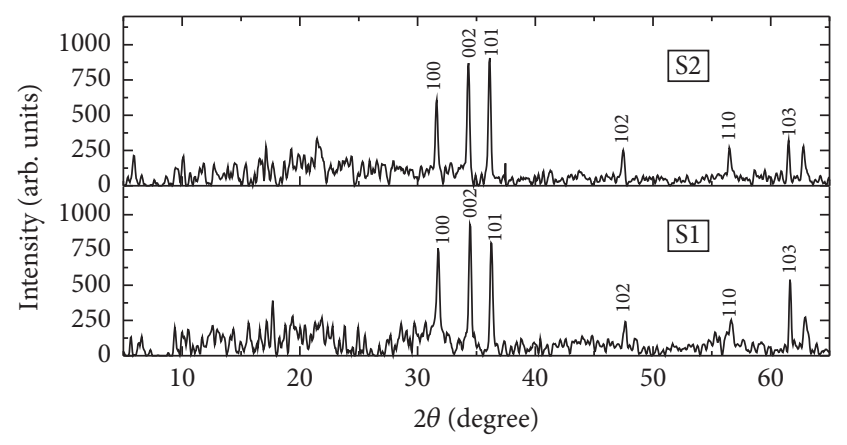

FIGURE 1: X-ray diffraction (XRD) patterns obtained for $\mathrm{ZnO}$ thin films S1 and S2.

reflection at (002) plane, which is the preferential orientation and the densest plane of this sample. S2 has the strongest reflection at (101), the preferential orientation and the densest plane of this sample. Reflections for planes (100), (002), (101), (102), (110), (103), and (112) are observed for both samples.

Top view scanning electron microscope images for S1 and S2 are depicted in Figures 2(a) and 2(b), respectively. The average crystallite size of S1 is $252 \mathrm{~nm}$ and that of S2 is $346 \mathrm{~nm}$, by measuring the size of 60 particles of S1 and S2 and obtaining the average of them. The increment in the size is attributed to the increment time in the heat treatment. While S1 was treated only one time at $950^{\circ} \mathrm{C}$ for one hour, S2 was treated two times at $950^{\circ} \mathrm{C}$, one hour for each treatment. The cross sectional view of S1 and S2 is displayed in Figures 2(c) and 2(d), respectively. From the images, the growth of S1 is better than S2. This is in agreement with the results of $\mathrm{X}$-ray diffraction in Figure 1. The thickness of S1 is about 2 micrometers. Since there is no continuous growth in S2 in the vertical direction, the thickness of S2 cannot be deduced from SEM image.

To generate room temperature ultraviolet stimulated emission using sol-gel spin coated $\mathrm{ZnO}$ thin films, many samples have been prepared with different conditions. The first condition that has been considered was the size of the crystallites. According to our previous work as well as the work considered by the other groups, the larger the crystallite size is, the larger the optical gain obtained from the material is, and thus the possibility of obtaining stimulated emission from the samples. The conditions that lead to larger crystallites of the film are treating the samples at higher temperature such that more growth for the crystallites occurs, increasing the film thickness by increasing the number of layers and increasing the initial concentration of the zinc salt. Optimum conditions have been searched to achieve larger crystallites with less material defects. According to the investigations that have been made, the following are the best conditions in our case that lead to larger crystallites with low defects. The optimum zinc precursor concentration was $0.75 \mathrm{M}$. Lower concentrations than this one lead to increasing the number of crystallites; however the sizes of these crystallites are small. On the other hand, higher concentration leads to porous structure. This is in agreement with the work published by O'Brien et al. [29]. The optimum film thickness was about 2.6 


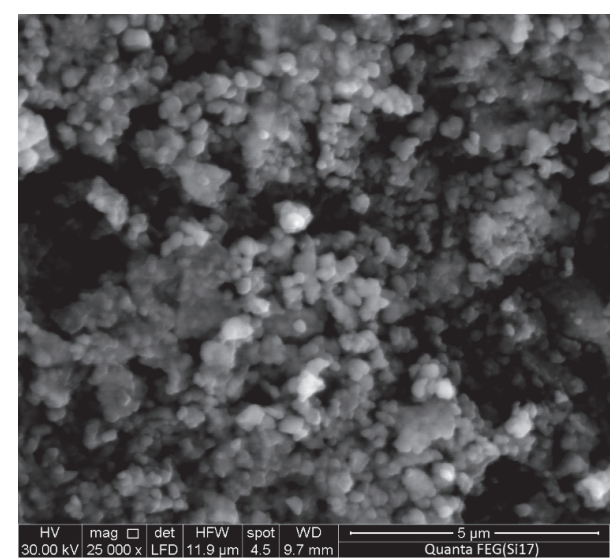

(a)
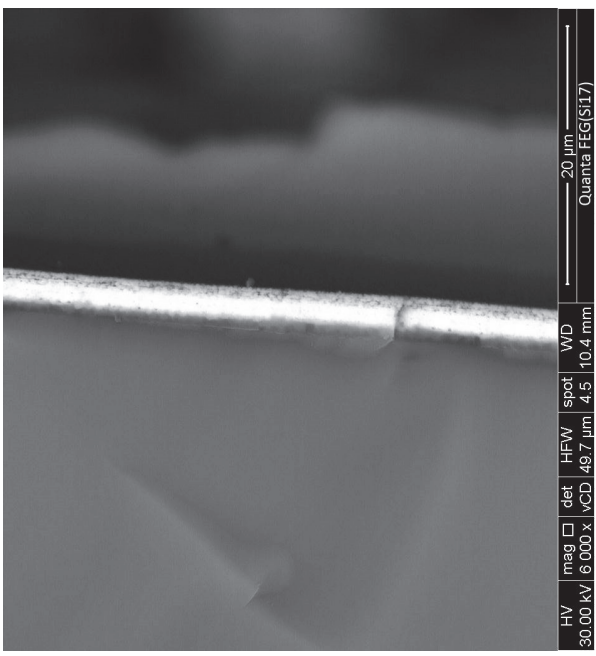

(c)

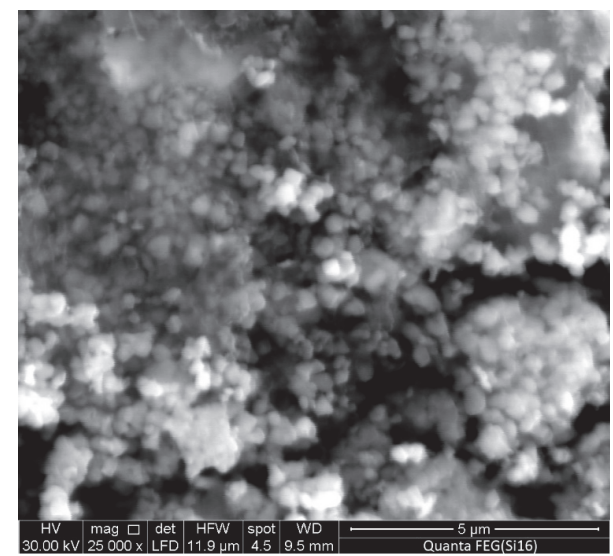

(b)

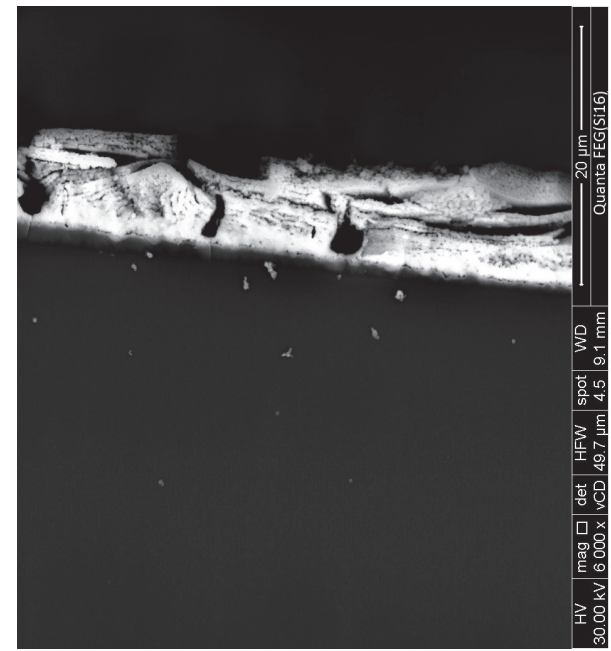

(d)

FIgure 2: Top view of $\mathrm{ZnO}$ thin films $\mathrm{S} 1$ and $\mathrm{S} 2$ using SEM.

micrometers, which was obtained by depositing 19 layers at initial zinc salt of $0.75 \mathrm{M}$, with 10-layer deposition followed by heat treatment of $575^{\circ} \mathrm{C}$ followed by deposition of another 9 layers and then the sample was heat treated at $950^{\circ} \mathrm{C}(\mathrm{S} 1)$. When another 11 layers was deposited, followed by another heat treatment (S2), cracking in the film was observed which is due to the third heat treatment and the thick film on silicon substrate, which has a mismatch in the lattice constant compared to that of $\mathrm{ZnO}$. Figure 3 shows the emission from the different samples at pumping intensity of $28.7 \mathrm{MW} / \mathrm{cm}^{2}$. For sample with only 10 layers treated at 575,750 , and $950^{\circ} \mathrm{C}$, spontaneous emission occurs with a peak at around $389 \mathrm{~nm}$ with weak emission intensity as in Figure 3. On the other hand, samples S1 and S2 show strong emission with narrow spectral width.

To investigate stimulated emission process from samples S1 and S2, input-output intensity dependence has been investigated. Figure 4 shows the results of input-output intensity dependence. At low pump intensity from 5 to about $23 \mathrm{MW} / \mathrm{cm}^{2}$, there is a linear relationship between the output and input intensity. The red line in the curve is to show such linear dependence. At higher intensity more than $23 \mathrm{MW} / \mathrm{cm}^{2}$, there is a deviation from such linear dependence, and the output intensity growth is superlinear. Further, spectral width gets decreased for intensity higher than $23 \mathrm{MW} / \mathrm{cm}^{2}$, as indicated in Figure 5. From Figures 4 and 5 stimulated emission was evident due to the deviation from linear relation between input-output intensity and the decrease in the spectral width with increasing the pump intensity. The threshold intensity, the input intensity at which the kink in the input-output intensity occurs, is $22.6 \mathrm{Mw} / \mathrm{cm}^{2}$ for sample S1 and $23.2 \mathrm{MW} / \mathrm{cm}^{2}$ for sample S2. The lower the threshold intensity for stimulated emission is, the better the quality of the sample is; hence S1 sample has better quality compared to S2 according to the results of the threshold values. This is in agreement with the results of X-ray measurements (depicted in Figure 1) and the results of cross sectional view of SEM images presented in Figure 2.

Figures 6(a) and 6(b) show the spectrum emitted from S1 and S2 at different input intensities. At lower pump intensities below the thresholds of S1 and S2, the photoluminescence emitted from the samples is weak and broad. These are characteristics for spontaneous emission. At higher pump intensities than threshold the emission is huge and with 


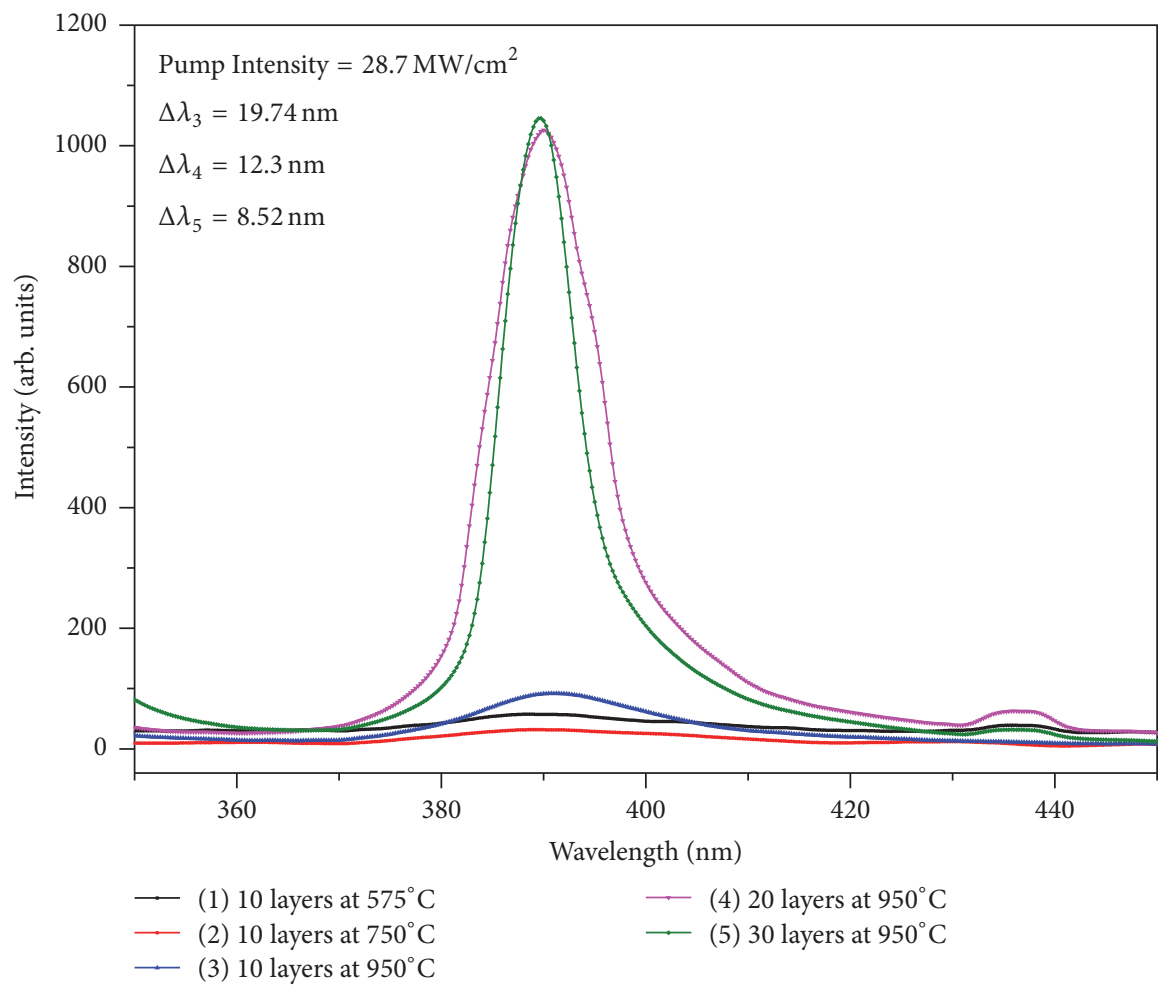

FIGURE 3: Progress of emission at different preparation conditions at pump intensity $\left[28.7 \mathrm{MW} / \mathrm{cm}^{2}\right]$.

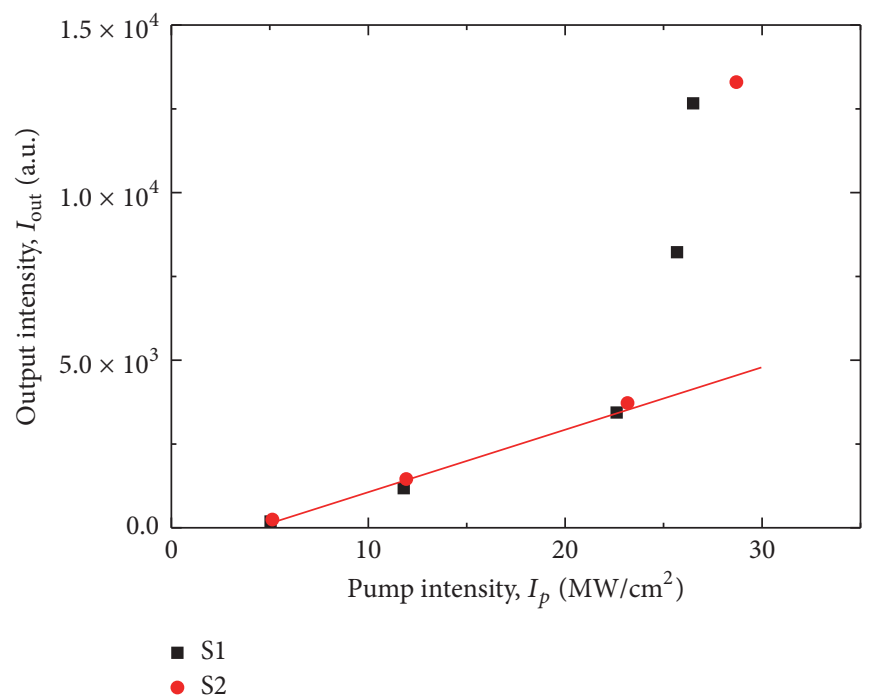

FIGURE 4: Input-output intensity dependence of S1 and S2.

narrow spectral width, which are the characteristics of stimulated emission.

There are several mechanisms by which stimulated emission can occur. It can occur by single pass gain through high gain $\mathrm{ZnO}$ thin films, exciton-exciton scattering with P-band lasing, electron-hole plasma, surface undulations, P-band lasing assisted with index guiding through topdown microstructuring, random lasing, exciton-polariton interaction, or by coherent reflections from parallel hexagons. In the single pass gain, a stripe has been focused to a high gain
$\mathrm{ZnO}$ thin films such that the spontaneous emission passes through highly inverted region, which leads to stimulated emission with high intensity. The stimulated emission occurs in the transverse direction relative to the exciting beam. In the exciton-exciton scattering P-band lasing, two excited excitons interact with each other such that one exciton is induced to emit radiation and the other gets excited to higher excitonic state. The stimulated emission intensity in this process is superlinear with the incident radiation with a power exponent as high as 8. There is approximately 


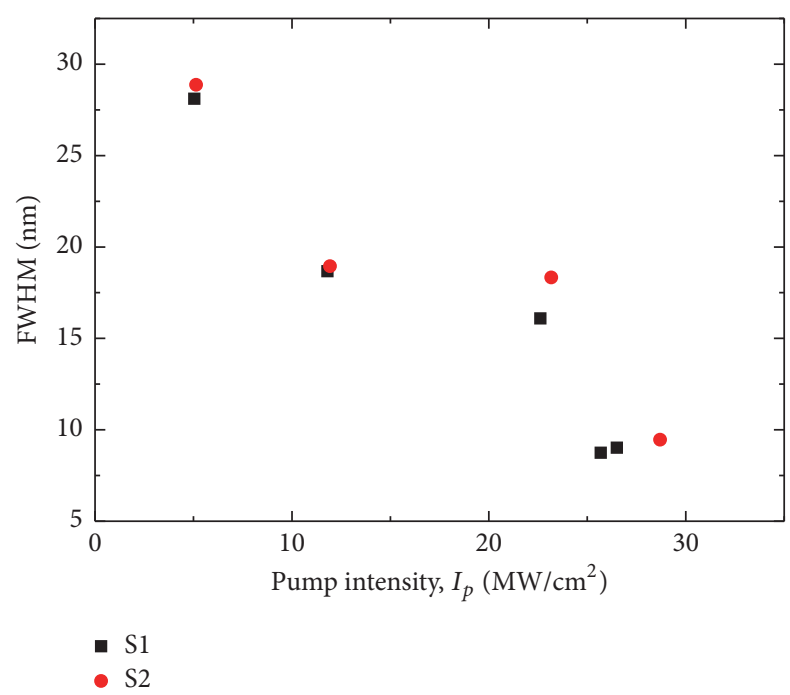

FIGURE 5: Spectral width at different pump intensities for S1 and S2.

no change in the emitted wavelength for P-band lasing as the incident intensity increases. For electron-hole plasma lasing, the density of excitons exceeds Mott density, and dissociations of excitons occur and electron-hole plasma is formed. Recombination between electrons and holes in this region of excitations leads to stimulated emission. With increasing the excitation intensity in the electronhole plasma region of excitation, the emitted wavelength increases considerably due to bandgap renormalization. For surface undulations mechanism, surface undulations cause scattering for the emitted photons in the surface which leads to stimulated emission. The emitted wavelength lies in the region of P-band. For surface undulations assisted with topdown microstructuring, in addition to the scattered photons by surface undulations that cause stimulated emission, the process is further improved via waveguiding caused by topdown microstructuring. The emitted wavelength is shifted towards longer wavelength relative to absence of microstructuring. For both random lasing and coherent reflections by aligned hexagons, several narrow band multipeaks occur in the spectrum of the emitted radiation. However, the cavity lengths for both mechanisms are different. For self-assembled hexagons the cavity length is the stripe length that excites the parallel hexagons, but for random lasing the cavity length is the pass length after which the photons return back to their original position. Gadallah et al. reported the mechanisms of stimulated emissions by single pass gain, P-band lasing, electron-hole plasma, and surface undulations [9]. Nomenyo et al. reported the mechanism of stimulated emission by surface undulation enhanced top-down microstructuring. [28]. Dupont reported the mechanism of random lasing [19]. These mechanisms can be distinguished from each other. For example, P-band lasing and electron-hole plasma lasing can be distinguished as follows. The emitted wavelength drastically gets longer as the input intensity increases in case of electron-hole plasma lasing. However, in case of Pband lasing there is approximately no change of the emitted wavelength. Random lasing is distinguished by narrow multiple peaks in the emitted spectra. Here, there is very small change in the emitted wavelength at different pump powers as indicated in Figure 7. In addition, the input-output intensity dependence in the stimulated emission region, the inset in Figure 7, shows a higher power exponent with an exponent of 8.2 for S1 and 5.9 for S2. Both the small change in the peak emission wavelength with increasing the pump intensity and the superlinear dependence of the output intensity versus the pump intensity with a power exponent greater than five indicate that the mechanism of stimulated emission is by exciton-exciton P-band.

Figure 8 depicts the quantum efficiency, defined as emitted photons/absorbed photons, as a function of the pump intensity for samples S1 and S2. With increasing the pump intensity, the quantum efficiency increases too. The highest value of the quantum efficiency for S1 is $24.2 \%$ and that of S2 is $23.5 \%$.

\section{Analysis}

The generated number of excitons per excited volume $n_{\mathrm{X}-\mathrm{X}}$ can be calculated using the following equation:

$$
n_{\mathrm{X}-\mathrm{X}}=\frac{N \tau_{\mathrm{ex}}}{V \tau_{p}} \gamma_{\mathrm{ph}} \gamma_{d}
$$

where $N$ is incident number of photons $=E / h v, E$ is the energy per pulse of the exciting laser, $h v$ is the energy of one photon, $V=A l$ is the excited volume, where $A$ is the excited area and $l$ is a characteristic length, $\gamma_{\mathrm{ph}}$ is the loss of the incident photons by nonabsorption process such as reflection, or presence of voids in the excited area, $\tau_{\mathrm{ex}}$ is the exciton lifetime, $\tau_{p}$ is the pulse duration of the exciting laser, and $\gamma_{d}$ is the defect loss such as surface-state defects or grain boundary defects. The process can be theoretically governed by the following:

$$
n_{\mathrm{X}-\mathrm{X}}=\frac{N \tau_{\mathrm{ex}}}{V \tau_{p}} \gamma_{\mathrm{ph}} \gamma_{d} \leq n_{\mathrm{Mott}}
$$

where $n_{\text {Mott }}$ is the so-called Mott density, which accounts for the following process: as the number of excitons increase more and more, Coulomb interactions between these excitons become strong that the excitons lose their individual nature and a new phase called electron-hole plasma arises. Further, the quantum efficiency plus the different losses should be equal to unity; that is,

$$
\eta_{\mathrm{Q} . \mathrm{E}}+\gamma_{\mathrm{ph}} \gamma_{d}+\gamma_{\mathrm{nr}}=1
$$

where $\gamma_{\mathrm{nr}}$ is the nonradiative recombination loss of the generated excitons by surface-state defects or scattering centers.

To quantify these parameters to our measurements, with a spot diameter of the laser beam of $0.6 \mathrm{~mm}$ on the film, $A=2.8 \times 10^{-3} \mathrm{~cm}^{2}, l \approx 200 \mathrm{~nm}$ (depth through which the process occurs, which could be of the order of the exciton diffusion length), $N=65 \mu \mathrm{J} /\left(6.626 \times 10^{-34} \times 3 \times 10^{10} / 337\right.$ $\left.\times 10^{-7}\right)=1.1 \times 10^{14}$ photons, $\tau_{\mathrm{ex}} / \tau_{p}=0.1 \mathrm{~ns} / 0.8 \mathrm{~ns}=0.125$ 


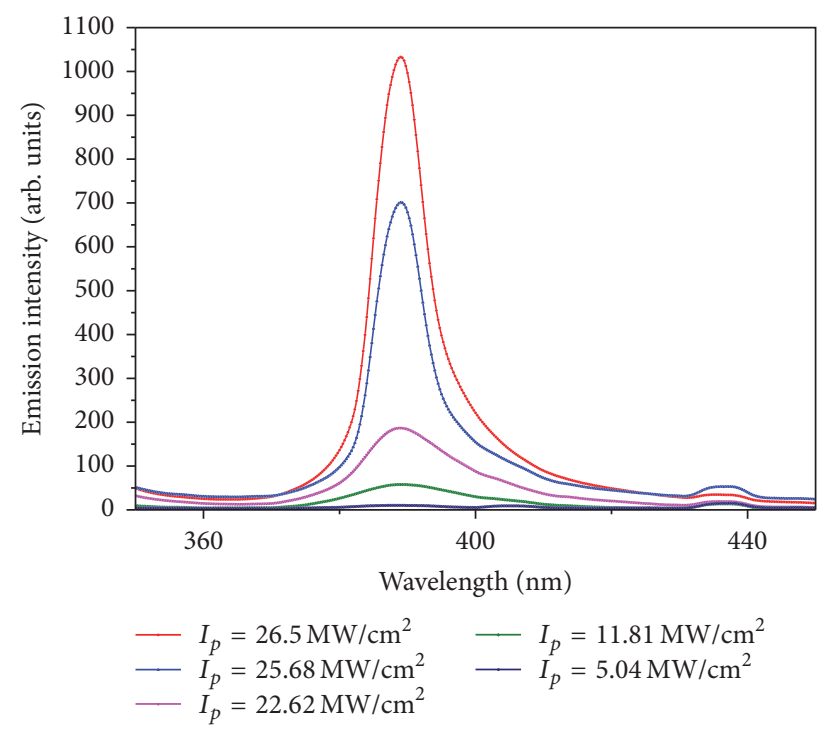

(a)

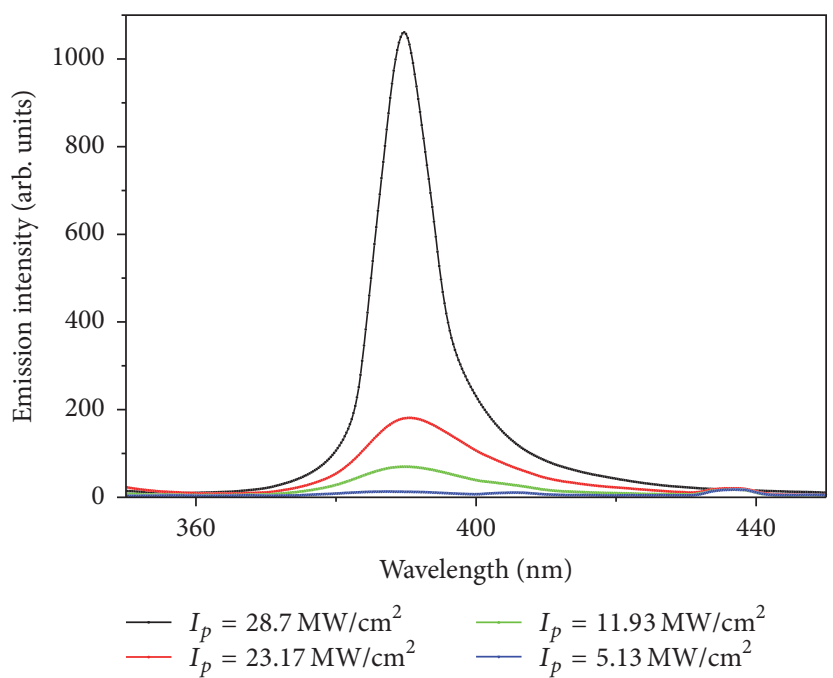

(b)

FIgURE 6: Spectra emitted from (a) S1 and (b) S2 at different pump intensities.

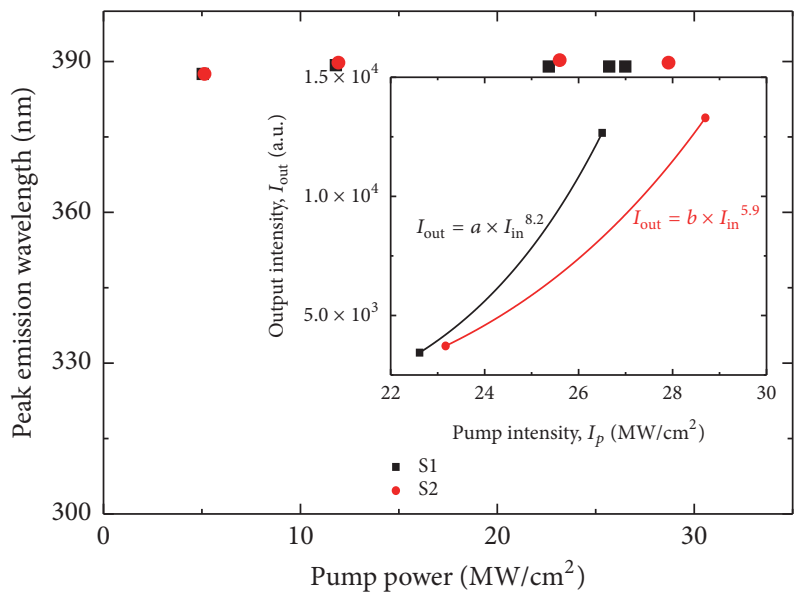

- $\mathrm{S} 1$

- S2

FIGURE 7: Peak emitted wavelength at different pump intensities for S1 and S2. Inset is the input-output intensity dependence in the stimulated emission region.

with $\tau_{\text {ex }}=0.1 \mathrm{~ns}$ as reported in [30], room temperature Mott density $n_{\text {Mott }} \approx 3.7 \times 10^{19}$ excitons $/ \mathrm{cm}^{3}$ as reported in [31], $\eta_{\text {Q.E }}=0.242$ (Figure 8 ), and $\gamma_{\text {ph }} \gamma_{d}$ is then less than or equal to 0.15 (using (2)). From (3), $\gamma_{\mathrm{nr}}=1-\eta_{\mathrm{Q} . \mathrm{E}}+\gamma_{\mathrm{ph}} \gamma_{d} \approx 0.608$.

\section{Conclusions}

Preparation of $\mathrm{ZnO}$ thin films by sol-gel spin coating on $\mathrm{n}^{+}$As-doped $\langle 100\rangle$ Si-substrate for the purpose of optically pumped stimulated emission has been reported. The optimum condition for the preparation of the film has been presented. The film thickness was about 2.6 micrometers.

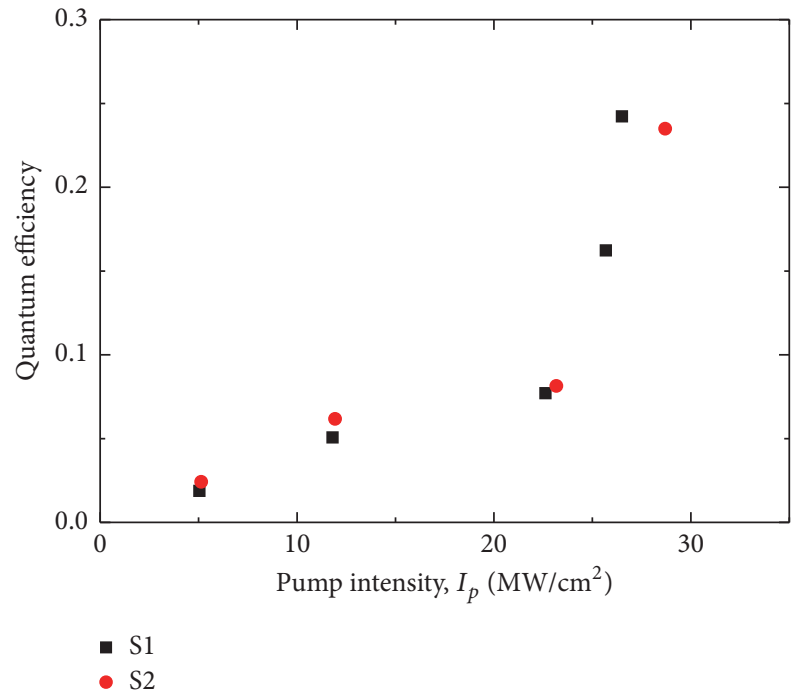

FIGURE 8: Quantum efficiency at different pump intensities for S1 and S2.

Two heat treatments were performed for better crystallization of the film, one at $575^{\circ} \mathrm{C}$, and the other at $950^{\circ} \mathrm{C}$. The mechanism responsible for stimulated emission is excitonexciton P-band lasing as there is a small shift in the peak emission wavelength and a power exponent larger than five in output intensity versus input intensity and there is no multiple peaks narrow band in the spectra of the stimulated emission. A quantum efficiency of about $24.2 \%$ has been achieved. The results show that sol-gel spin coating is a promising way for the preparation of $\mathrm{ZnO}$ thin films that can generate low cost ultraviolet stimulated emission. 


\section{Conflicts of Interest}

The authors declare that they have no conflicts of interest.

\section{References}

[1] G. Sharma, "Review of applications of optics in health care and life sciences," Research Journal of Chemical \& Environmental Sciences, vol. 4, article 7, 2016.

[2] F. J. Manzano-Moreno, R. Medina-Huertas, J. Ramos-Torrecillas, O. García-Martínez, and C. Ruiz, "The effect of lowlevel diode laser therapy on early differentiation of osteoblast via BMP-2/TGF- $\beta 1$ and its receptors," Journal of CranioMaxillofacial Surgery, vol. 43, no. 9, pp. 1926-1932, 2015.

[3] R. R. Anderson, "Lasers in dermatology—a critical update," The Journal of Dermatology, vol. 27, no. 11, pp. 700-705, 2000.

[4] J. E. Schoenly, W. Seka, J. D. B. Featherstone, and P. Rechmann, "Near-UV laser treatment of extrinsic dental enamel stains," Lasers in Surgery and Medicine, vol. 44, no. 4, pp. 339-345, 2012.

[5] Ü. Özgür, D. Hofstetter, and H. Morkoç, "ZnO devices and applications: a review of current status and future prospects," Proceedings of the IEEE, vol. 98, no. 7, pp. 1255-1268, 2010.

[6] A. S. Matharu, S. Jeeva, and P. S. Ramanujam, "Liquid crystals for holographic optical data storage," Chemical Society Reviews, vol. 36, no. 12, pp. 1868-1880, 2007.

[7] S. A. Slattery, D. N. Nikogosyan, and G. Brambilla, "Fiber Bragg grating inscription by high-intensity femtosecond UV laser light: comparison with other existing methods of fabrication," Journal of the Optical Society of America B: Optical Physics, vol. 22, no. 2, pp. 354-361, 2005.

[8] R. W. Nesbitt, T. Hirata, I. B. Butler, and J. A. Milton, "UV laser ablation ICP-MS: some applications in the earth sciences," Geostandards Newsletter: The Journal of Geostandards and Geoanalysis, vol. 21, no. 2, pp. 231-243, 1997.

[9] A.-S. Gadallah, K. Nomenyo, C. Couteau, D. J. Rogers, and G. Lérondel, "Stimulated emission from $\mathrm{ZnO}$ thin films with high optical gain and low loss," Applied Physics Letters, vol. 102, no. 17, Article ID 171105, 2013.

[10] D. Horwat, M. Mickan, and W. Chamorro, "New strategies for the synthesis of $\mathrm{ZnO}$ and $\mathrm{Al}$-doped $\mathrm{ZnO}$ films by reactive magnetron sputtering at room temperature," Physica Status Solidi C, Current Topics in Solid State Physics, vol. 13, no. 10-12, pp. 951-957, 2016.

[11] S.-Y. Kuo, F.-I. Lai, W.-C. Chen, C.-P. Cheng, H.-C. Kuo, and S.-C. Wang, "Ultraviolet lasing of sol-gel-derived zinc oxide polycrystalline films," Japanese Journal of Applied Physics, vol. 45, no. 4, pp. 3662-3665, 2006.

[12] A. Gadallah and M. M. El-Nahass, "Structural, optical constants and photoluminescence of $\mathrm{ZnO}$ thin films grown by sol-gel spin coating," Advances in Condensed Matter Physics, vol. 2013, Article ID 234546, 11 pages, 2013.

[13] A. Gadallah, "Fabrication and characterization of solution processed vertically aligned $\mathrm{ZnO}$ microrods," Applied Surface Science, vol. 311, pp. 172-176, 2014.

[14] S. S. Badadhe and I. Mulla, "Effect of aluminium doping on structural and gas sensing properties of zinc oxide thin films deposited by spray pyrolysis," Sensors and Actuators B: Chemical, vol. 156, no. 2, pp. 943-948, 2011.

[15] Y. W. Heo, D. P. Norton, and S. J. Pearton, "Origin of green luminescence in $\mathrm{ZnO}$ thin film grown by molecular-beam epitaxy," Journal of Applied Physics, vol. 98, no. 7, Article ID 073502, 2005.
[16] S. T. Tan, B. J. Chen, X. W. Sun et al., "Blueshift of optical band gap in $\mathrm{ZnO}$ thin films grown by metal-organic chemical-vapor deposition," Journal of Applied Physics, vol. 98, no. 1, Article ID 013505, 2005.

[17] C.-X. Wang, C.-Y. Lv, C. Zhu et al., "Electrically pumped random lasing with an onset voltage of sub-3 $\mathrm{V}$ from $\mathrm{ZnO}$ based light-emitting devices featuring nanometer-thick $\mathrm{MoO}_{3}$ interlayers," Nanoscale, vol. 7, no. 20, pp. 9164-9168, 2015.

[18] C. Wang, C. Zhu, C. Lv, D. Li, X. Ma, and D. Yang, "Electrically pumped random lasing from hydrothermal $\mathrm{ZnO}$ films of large grains," Applied Surface Science, vol. 332, pp. 620-624, 2015.

[19] P. Dupont, C. Couteau, D. J. Rogers, F. Hosseini Téhérani, and G. Lérondel, "Waveguiding-assisted random lasing in epitaxial $\mathrm{ZnO}$ thin film," Applied Physics Letters, vol. 97, no. 26, Article ID 261109, 2010.

[20] Z. K. Tang, M. Kawasaki, A. Ohtomo, H. Koinuma, and Y. Segawa, "Self-assembled $\mathrm{ZnO}$ nano-crystals and exciton lasing at room temperature," Journal of Crystal Growth, vol. 287, no. 1, pp. 169-179, 2006.

[21] C. Chia and M. Yen, "Radiative efficiency of inelastic excitonexciton scattering in $\mathrm{ZnO}$ nanocrystalline films," Optical Materials Express, vol. 4, no. 5, article 1023, 2014.

[22] P. Zu, A. Ohtomo, Y. Segawa et al., "Ultraviolet spontaneous and stimulated emissions from $\mathrm{ZnO}$ microcrystallite thin films at room temperature," Solid State Communications, vol. 103, no. 8, pp. 459-463, 1997.

[23] J. Dai, C. X. Xu, X. Y. Xu, J. T. Li, J. Y. Guo, and Y. Lin, "Controllable fabrication and optical properties microdisk for whispering gallery mode microlaser," APL Materials, vol. 1, Article ID 032105, 2013.

[24] T. Nobis, E. M. Kaidashev, A. Rahm, M. Lorenz, and M. Grundmann, "Whispering gallery modes in nanosized dielectric resonators with hexagonal cross section," Physical Review Letters, vol. 93, no. 10, Article ID 103903, 2004.

[25] J. Wiersing, "Hexagonal dielectric resonators and microcrystal lasers," Physical Review A, vol. 67, no. 2, Article ID 023807, 12 pages, 2003.

[26] S. M. Spillane, T. J. Kippenberg, and K. J. Vahala, "Ultralowthreshold Raman laser using a spherical dielectric microcavity," Nature, vol. 415, no. 6872, pp. 621-623, 2002.

[27] X. Q. Zhang, I. Suemune, H. Kumano, J. Wang, and S. H. Huang, "Surface-emitting stimulated emission in high-quality $\mathrm{ZnO}$ thin films," Journal of Applied Physics, vol. 96, no. 7, pp. 3733-3736, 2004.

[28] K. Nomenyo, A. Gadallah, S. Kostcheev, D. J. Rogers, and G. Lérondel, "Enhanced stimulated emission in $\mathrm{ZnO}$ thin films using microdisk top-down structuring," Applied Physics Letters, vol. 104, no. 18, Article ID 181104, 2014.

[29] S. O’Brien, L. H. K. Koh, and G. M. Crean, “ZnO thin films prepared by a single step sol-gel process," Thin Solid Films, vol. 516, no. 7, pp. 1391-1395, 2008.

[30] T. Nakamura, K. Firdaus, and S. Adachi, "Electron-hole plasma lasing in a $\mathrm{ZnO}$ random laser," Physical Review B, vol. 86, no. 20, Article ID 205103, 2012.

[31] B. J. Kim and K. Kyhm, "Optical modal gain saturation of exciton-exciton scattering and electron-hole plasma in $\mathrm{ZnO}$," Journal of the Korean Physical Society, vol. 51, no. 5, pp. 17261731, 2007. 

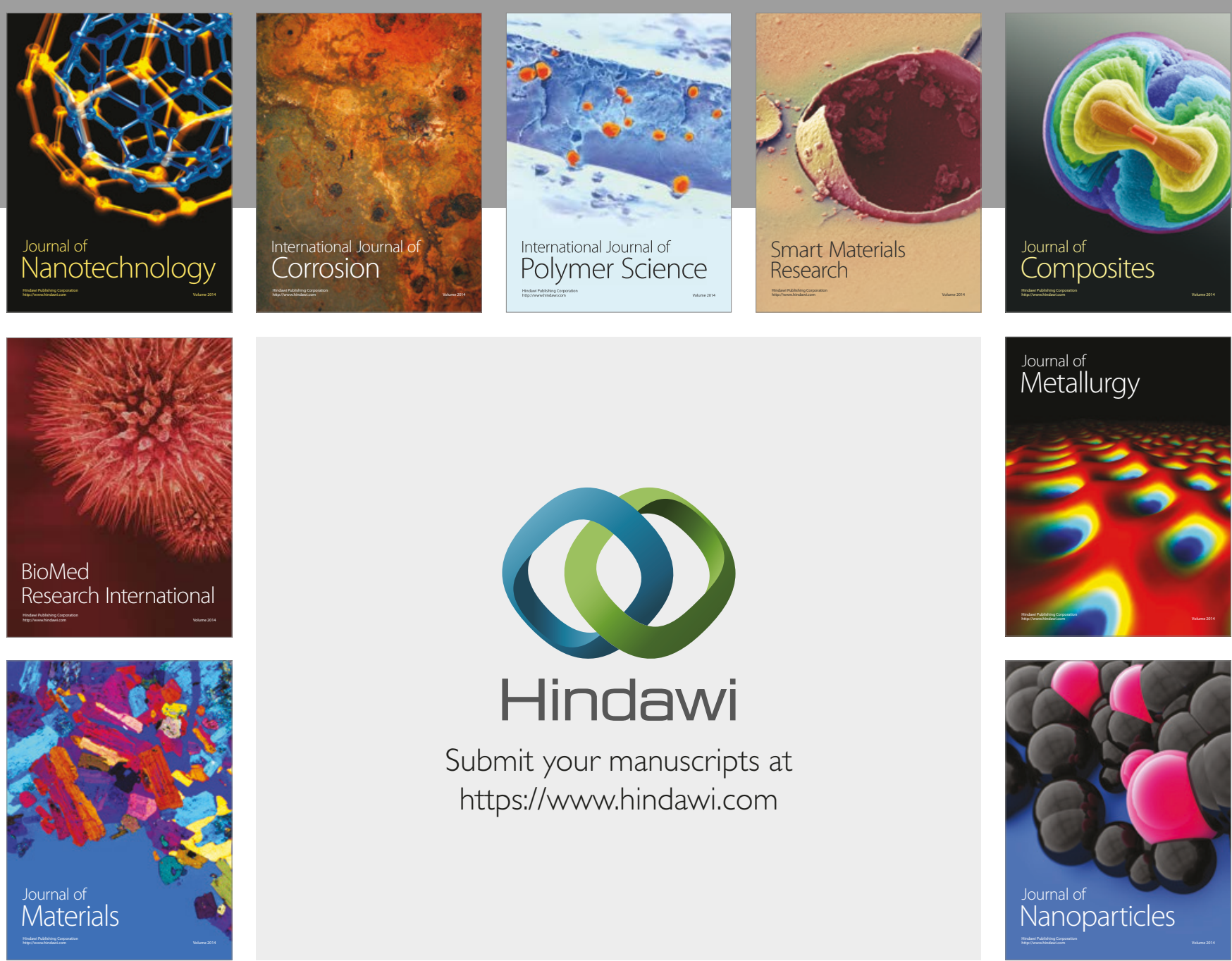

\section{Hindawi}

Submit your manuscripts at

https://www.hindawi.com
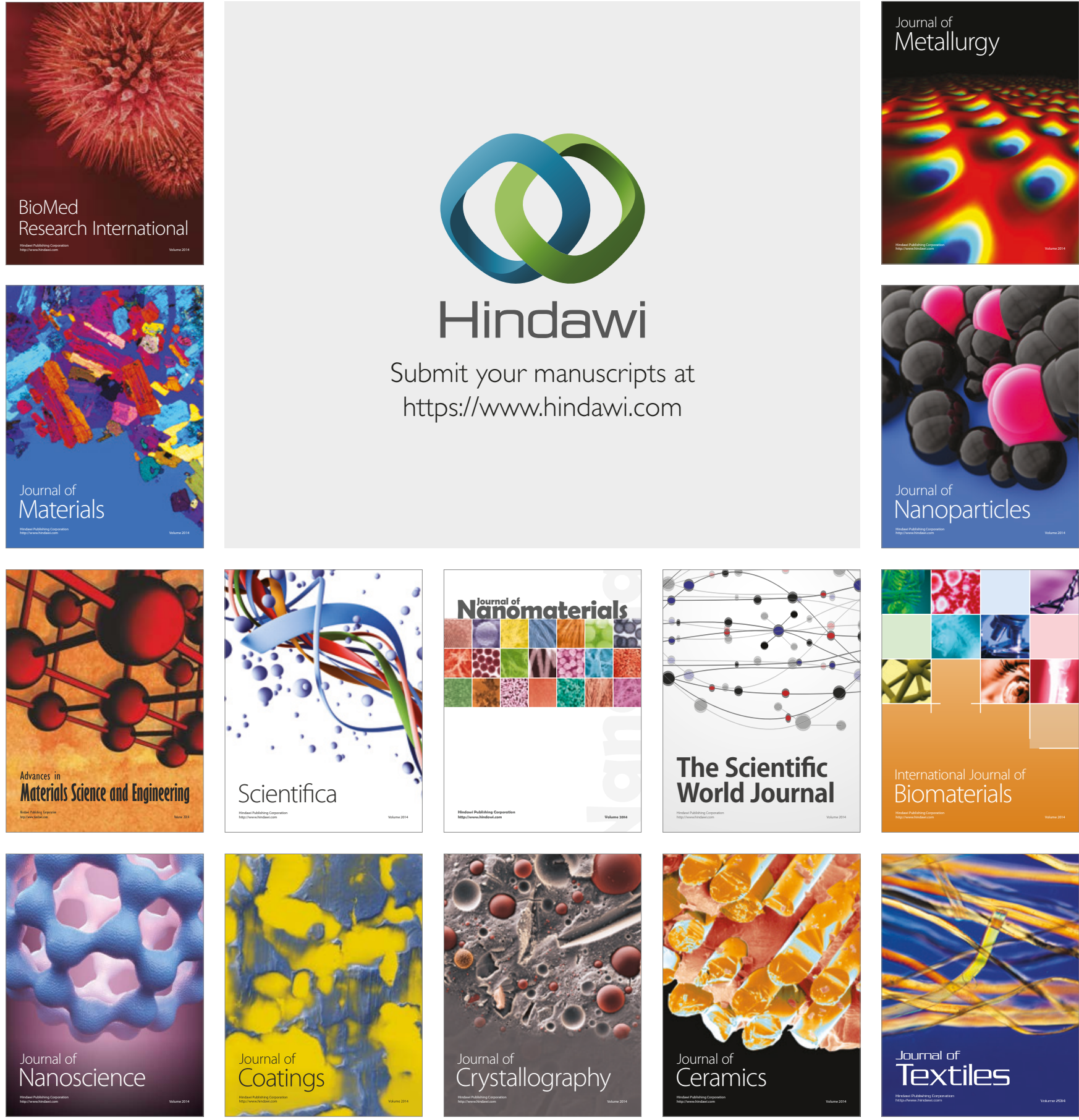

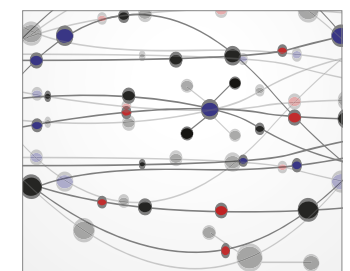

The Scientific World Journal
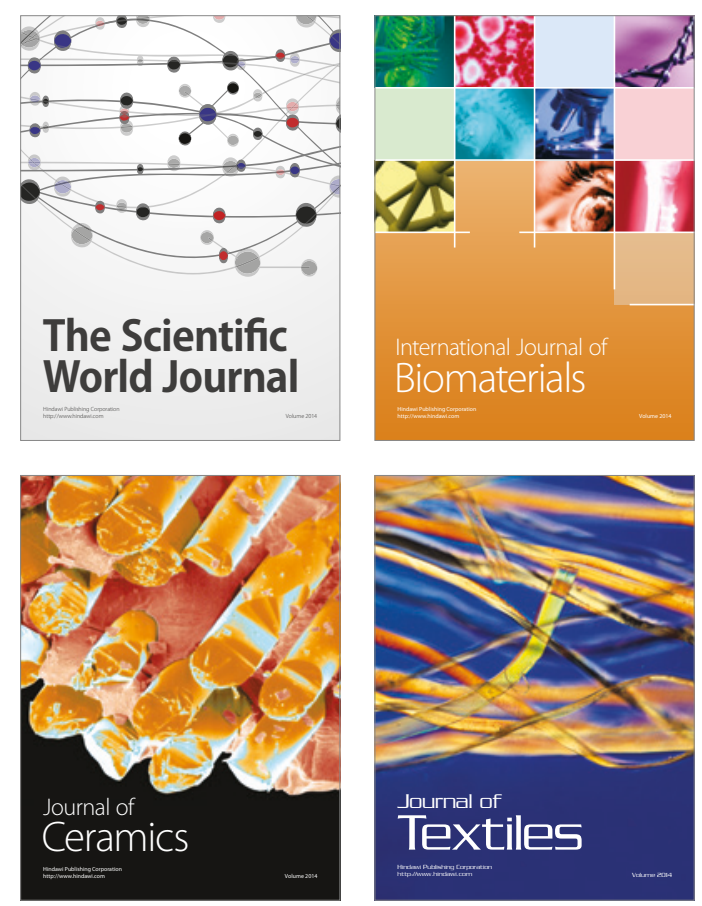